\title{
Homeotopy groups of leaf spaces of one-dimensional foliations on non-compact surfaces with non-compact leaves
}

\author{
Sergiy Maksymenko, Eugene Polulyakh
}

\begin{abstract}
Let $Z$ be a non-compact two-dimensional manifold obtained from a family of open strips $\mathbb{R} \times(0,1)$ with boundary intervals by gluing those strips along some pairs of their boundary intervals. Every such strip has a natural foliation into parallel lines $\mathbb{R} \times t, t \in(0,1)$, and boundary intervals which gives a foliation $\Delta$ on all of $Z$. Denote by $\mathcal{H}(Z, \Delta)$ the group of all homeomorphisms of $Z$ that maps leaves of $\Delta$ onto leaves and by $\mathcal{H}(Z / \Delta)$ the group of homeomorphisms of the space of leaves endowed with the corresponding compact open topologies. Recently, the authors identified the homeotopy group $\pi_{0} \mathcal{H}(Z, \Delta)$ with a group of automorphisms of a certain graph $G$ with additional structure which encodes the combinatorics of gluing $Z$ from strips. That graph is in a certain sense dual to the space of leaves $Z / \Delta$.

On the other hand, for every $h \in \mathcal{H}(Z, \Delta)$ the induced permutation $k$ of leaves of $\Delta$ is a homeomorphism of $Z / \Delta$ and the correspondence $h \mapsto k$ is a homomorphism $\psi: \mathcal{H}(\Delta) \rightarrow \mathcal{H}(Z / \Delta)$. The aim of the present paper is to show that $\psi$ induces a homomorphism of the corresponding homeotopy groups $\psi_{0}: \pi_{0} \mathcal{H}(Z, \Delta) \rightarrow \pi_{0} \mathcal{H}(Z / \Delta)$ which turns out to be either injective or having a kernel $\mathbb{Z}_{2}$. This gives a dual description of $\pi_{0} \mathcal{H}(Z, \Delta)$ in terms of the space of leaves.
\end{abstract}

Анотація. Нехай некомпактний двовимірний многовид $Z$ отримано 3 сім'ї відкритих смуг $\mathbb{R} \times(0,1)$, що мають на межі відкриті інтервали, за допомогою склеювання цих смуг вздовж деяких пар їх межових інтервалів. Кожна така смуга має природне шарування, шарами якого $є$ паралельні прямі $\mathbb{R} \times t, t \in(0,1)$, а також межові інтервали. Ці шарування породжують однозначно визначене шарування $\Delta$ на $Z$. Позначимо

Keywords: foliation, leaf space, striped surface

Ключові слова: шарування, простір шарів, смугаста поверхня

DOI: http://dx.doi.org/10.15673/tmgc.v14i4.2204 
через $\mathcal{H}(Z, \Delta)$ групу гомеоморфізмів $Z$, які відображають шари шарування $\Delta$ на шари, а через $\mathcal{H}(Z / \Delta)$ - групу гомеоморфізмів простору шарів. Наділимо ці групи компактно-відкритими топологіями. Нещодавно автори ідентифікували групу гомеотопій $\pi_{0} \mathcal{H}(Z, \Delta)$ з групою автоморфізмів певного графу $G$ з додатковою структурою, яка кодує комбінаторику склеювання $Z$ зі смуг. Вказаний граф є в деякому сенсі дуальним до простору шарів $Z / \Delta$.

3 іншого боку, для кожного $h \in \mathcal{H}(Z, \Delta)$ індукована перестановка $k$ шарів $\Delta \in$ гомеоморфізмом $Z / \Delta$, а відповідність $h \mapsto k \in$ гомоморфізмом $\psi: \mathcal{H}(\Delta) \rightarrow \mathcal{H}(Z / \Delta)$. У даній роботі показано, що $\psi$ індукує гомоморфізм відповідних груп гомеотопій $\psi_{0}: \pi_{0} \mathcal{H}(Z, \Delta) \rightarrow \pi_{0} \mathcal{H}(Z / \Delta)$, причому він або ін'єктивний, або його ядро ізоморфне $\mathbb{Z}_{2}$. Це дає дуальний опис $\pi_{0} \mathcal{H}(Z, \Delta)$ у термінах простору шарів.

\section{INTRODUCTION}

In the present paper we study foliations on non-compact surfaces similar to foliations on the plane (considered by W. Kaplan [12,13], see also [6,7]) with "sufficiently regular behavior". Those foliations are studied in a series of papers by S. Maksymenko, Ye. Polulyakh, and Yu. Soroka, see [16-19, $21,23,24]$. Our main result (Theorem 1.4) relates the groups of isotopy classes of foliated (i.e. sending leaves to leaves) self-homeomorphisms of such surfaces with the groups of isotopy classes of homeomorphisms of the corresponding space of leaves (being in those cases non-Hausdorff onedimensional manifolds). This extends results by Yu. Soroka [24].

Let $Z$ be a surface endowed with a one-dimensional foliation $\Delta$. It will be convenient to say that the pair $(Z, \Delta)$ is a foliated surface. For an open subset $U \subset Z$ denote by $\left.\Delta\right|_{U}$ the induced foliation on $U$ consisting of connected components of non-empty intersections $\omega \cap U$ for all $\omega \in \Delta$.

Let $Y=Z / \Delta$ be the set of all leaves, and $p: Z \rightarrow Y$ be the natural projection associating to each $x \in Z$ the leaf of $\Delta$ containing $x$. Endow $Y$ with a quotient topology, so a subset $A \subset Y$ is open if and only if $p^{-1}(A)$ is open in $Z$. Notice that usually spaces of leaves of foliations are non-Hausdorff.

By sthe saturation $\operatorname{Sat}(U)$ of a subset $U \subset Z$ we mean the union of all leaves of $\Delta$ intersecting $U$. Evidently $\operatorname{Sat}(U)=p^{-1}(p(U))$. A subset $U \subset Z$ is called saturated (with respect to a foliation $\Delta$ ) whenever $U=\operatorname{Sat}(U)$.

Let $K$ be a one-dimensional manifold. Then by a trivial foliation on $\mathbb{R} \times K$ we will mean a foliation by lines $\{\mathbb{R} \times y\}_{y \in K}$.

A homeomorphism $h: Z \rightarrow Z$ is said to be leaf-preserving if $h(\omega)=\omega$ for each leaf $\omega \in \Delta$. Also, a homeomorphism $h: Z_{1} \rightarrow Z_{2}$ between foliated surfaces $\left(Z_{1}, \Delta_{1}\right)$ and $\left(Z_{2}, \Delta_{2}\right)$ is foliated if $h(\omega) \in \Delta_{2}$ for each $\omega \in \Delta_{1}$. In particular, every leaf-preserving homeomorphism is foliated. 
Let $\mathcal{H}(Z, \Delta)$ be the group of all foliated self-homeomorphisms of $Z$ and $\mathcal{H}(Y)$ be the group of homeomorphisms of $Y$ endowed with the corresponding compact open topologies. Let also $\mathcal{H}_{\text {id }}(Z, \Delta)$ be the identity path component of $\mathcal{H}(Z, \Delta)$ consisting of all homeomorphisms of $Z$ isotopic to $\operatorname{id}_{Z}$ in $\mathcal{H}(Z, \Delta)$, and $\mathcal{H}_{\text {id }}(Y)$ be the identity path component of $\mathcal{H}(Y)$. Evidently, $\mathcal{H}_{\text {id }}(Z, \Delta)$ and $\mathcal{H}_{\text {id }}(Y)$ are normal subgroups of the corresponding groups $\mathcal{H}(Z, \Delta)$ and $\mathcal{H}(Y)$ with respect to composition operation. Then the quotients

$$
\pi_{0} \mathcal{H}(Z, \Delta) \cong \mathcal{H}(Z, \Delta) / \mathcal{H}_{\mathrm{id}}(Z, \Delta), \quad \pi_{0} \mathcal{H}(Y) \cong \mathcal{H}(Y) / \mathcal{H}_{\mathrm{id}}(Y),
$$

i.e. the groups of path components of $\mathcal{H}(Z, \Delta)$ and $\mathcal{H}(Y)$, are called homeotopy groups of the foliation $\Delta$ and $Y$ respectively.

Notice that by definition each $h \in \mathcal{H}(Z, \Delta)$ induces a permutation of leaves of $\Delta$, and therefore it induces a bijection $k: Y \rightarrow Y$ making commutative the following diagram:

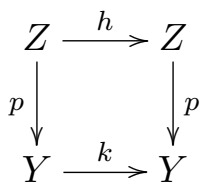

One easily checks that $k$ is a homeomorphism of $Y$ and that the correspondence $h \mapsto k$ is a homomorphism of groups

$$
\psi: \mathcal{H}(Z, \Delta) \rightarrow \mathcal{H}(Y), \quad \psi(h)=k .
$$

Definition 1.1. We will say that a foliated surface $(Z, \Delta)$ belongs to the class $\mathcal{F}$, whenever it satisfies the following conditions:

(A1) $Z$ has a countable base;

(A2) every leaf $\omega$ of $\Delta$ is a non-compact closed subset of $Z$, so it is an image of $\mathbb{R}$ under some topological embedding $\mathbb{R} \subset Z$;

(A3) each boundary component of $Z$ is a leaf of $\Delta$.

Our aim is to show (Theorem 1.4) that for foliations from class $\mathcal{F}$ satisfying certain additional "local finiteness" condition we have that

$$
\psi\left(\mathcal{H}_{\text {id }}(Z, \Delta)\right)=\mathcal{H}_{\text {id }}(Y) .
$$

This will imply that the image of $\psi$ is a union of path components of $\mathcal{H}(Y)$ and that $\psi$ induces a homomorphism of the corresponding homeotopy groups:

$$
\psi_{0}: \pi_{0} \mathcal{H}(Z, \Delta)=\frac{\mathcal{H}(Z, \Delta)}{\mathcal{H}_{\mathrm{id}}(Z, \Delta)} \longrightarrow \frac{\mathcal{H}(Y)}{\mathcal{H}_{\mathrm{id}}(Y)}=\pi_{0} \mathcal{H}(Y)
$$


Moreover, we also show that in this case for connected $Z$ the kernel of $\psi_{0}$ is either trivial or is isomorphic with $\mathbb{Z}_{2}$.

Definition 1.2. Let $(Z, \Delta)$ be a foliated surface from class $\mathcal{F}$.

1) Say that a leaf $\omega \in \Delta$ is regular if there exists an open saturated neighborhood $U$ of $\omega$ such that the pair $(\bar{U}, U)$ is "trivially foliated" in the sense that $(\bar{U}, U)$ is foliated homeomorphic

- either to the pair $(\mathbb{R} \times[-1,1], \mathbb{R} \times(-1,1))$ if $\omega \subset \operatorname{Int}(Z)$,

- or to the pair $(\mathbb{R} \times[0,1], \mathbb{R} \times[0,1))$ if $\omega \subset \partial Z$,

with trivial foliation into lines $\mathbb{R} \times t$, via a homeomorphism sending $\omega$ to $\mathbb{R} \times 0$.

2) A leaf which is not regular will be called singular'.

3) For a leaf $\omega$ define its Hausdorff closure by

$$
\operatorname{hcl}(\omega):=\underset{U \text { is a neighborhood of } \omega}{\overline{\operatorname{Sat}(U)}},
$$

so it is the intersection of closures of all saturated neighborhoods of $\omega$. Evidently, $\operatorname{hcl}(\omega)$ is saturated and $\omega \subset \operatorname{hcl}(\omega)$. We will say that $\omega$ is special whenever $\operatorname{hcl}(\omega) \backslash \omega \neq \varnothing$.

Denote by $\Delta_{\text {reg }}, \Delta_{\text {spec }}$, and $\Delta_{\text {sing }}$ respectively the families of all regular, special and singular leaves of $\Delta$. Then we have the following relations:

$$
\Delta_{\text {spec }} \subset \Delta_{\text {sing }}, \quad \Delta=\Delta_{\text {reg }} \sqcup \Delta_{\text {sing }}
$$

Remark 1.3. Notice that by definition each one-dimensional foliation $\Delta$ on a surface $Z$ is "locally regular", i.e. every point $x \in Z$ has a neighborhood $U$ such that the leaf of $\left.\Delta\right|_{U}$ containing $x$ is regular in the sense of Definition 1.2. Thus Definition 1.2 puts restriction to a global structure of the foliation near a leaf.

Let us also mention that it is possible that a leaf might have a trivially foliated saturated neighborhood $U$, however for any such neighborhood the pair $(\bar{U}, U)$ will never be trivially foliated. For example, let $\Delta=\{\mathbb{R} \times y\}_{y \in \mathbb{R}}$ be a trivial foliation on $\mathbb{R}^{2}$ into horizontal lines and $Z=\mathbb{R}^{2} \backslash 0$. Then it is easy to see that $\left.\Delta\right|_{Z}$ has two singular leaves $\omega_{1}=(-\infty ; 0) \times 0$ and $\omega_{2}=(0 ;+\infty) \times 0$. Indeed, any open saturated neighborhood $U$ of either of $\omega_{1}$ or $\omega_{2}$ contains $\mathbb{R} \times(-\varepsilon, \varepsilon) \backslash(0,0)$ for some small $\varepsilon>0$, and therefore $(\bar{U}, U)$ can not be foliated homeomorphic with $(\mathbb{R} \times[-1,1], \mathbb{R} \times(-1,1))$. This implies $\operatorname{hcl}\left(\omega_{1}\right)=\operatorname{hcl}\left(\omega_{2}\right)=\omega_{1} \cup \omega_{2}$, whence both $\omega_{1}$ and $\omega_{2}$ are special.

\footnotetext{
${ }^{1}$ There is some abuse of terminology used in [16, Property (c3) before Lemma 3.2]. Such leaves for striped surfaces were called special.
} 
The main result of the present paper is the following theorem relating the groups $\pi_{0} \mathcal{H}(Z, \Delta)$ and $\pi_{0} \mathcal{H}(Y)$.

Theorem 1.4. Let $(Z, \Delta)$ be a foliated surface from class $\mathcal{F}$ satisfying the following additional condition:

(A4) the family $\Delta_{\text {sing }}$ of all singular leaves of $\Delta$ is locally finite.

Then $\psi\left(\mathcal{H}_{\mathrm{id}}(Z, \Delta)\right)=\mathcal{H}_{\mathrm{id}}(Y)$, whence

(1) the image $\psi\left(\mathcal{H}_{\mathrm{id}}(Z, \Delta)\right)$ of the homomorphism $\psi: \mathcal{H}(Z, \Delta) \rightarrow \mathcal{H}(Y)$, see (1.2), is a union of path components of $\mathcal{H}(Y)$;

(2) $\psi$ induces a homomorphism $\psi_{0}: \pi_{0} \mathcal{H}(Z, \Delta) \rightarrow \pi_{0} \mathcal{H}(Y)$.

Moreover, if $Z$ is connected, then the kernel ker $\psi_{0}$ is either

(a) trivial, i.e. $\psi_{0}$ is injective, or

(b) ker $\psi_{0} \cong \mathbb{Z}_{2}$, which happens if and only if $\mathcal{H}(Z, \Delta)$ contains a leafpreserving homeomorphism which reverses orientation of all leaves.

In fact in a series of papers by the authors it was obtained a characterization of foliated surfaces $(Z, \Delta)$ from class $\mathcal{F}$ satisfying also the assumption (A4) of Theorem 1.4. It is shown in [20, Theorem 4.4] that every such surface is glued from certain "model strips" foliated by parallel lines. Such surfaces were called striped, and the proof of Theorem 1.4 essentially exploits an existence of that gluing. This description is motivated by results of W. Kaplan, see Theorem 3.1.5 below, describing the structure of foliations on $\mathbb{R}^{2}$.

Furthermore, in the joint paper with Yu. Soroka [21] the homeotopy group $\pi_{0} \mathcal{H}(Z, \Delta)$ of canonical foliation $\Delta$ of a striped surface $Z$ is identified with the group of automorphisms of a certain graph $G$ with additional structure encoding the gluing of $Z$ from model strips. In fact, the vertices of $G$ are strips and the edges are "seams" (i.e. leaves along which we glue the strips), so this graph is in a certain sense dual to the space of leaves $Y$. It is also proved in S. Maksymenko and O. Nikitchenko [15] that $G$ (as a one-dimensional CW-complex) is homotopy equivalent to the surface $Z$.

Thus Theorem 1.4 also gives a dual description of the homeotopy group $\pi_{0} \mathcal{H}(Z, \Delta)$ in terms of the space of leaves.

Structure of the paper. In Section 2 we consider several properties of non-Hausdorff $T_{1}$-spaces. In particular, we recall the exponential law for them as well as describe the relations between isotopies and paths in the groups of homeomorphisms for such spaces, see Corollary 2.1.2. We also present a characterization of the identity path component of the group of homeomorphisms of second countable $T_{1}$-manifolds that are not necessarily Hausdorff, see Lemma 2.4.1. In Section 3 we recall the results about striped surfaces, and prove Theorem 1.4 in Section 4. 


\section{2. $T_{1}$-SPACES}

We will recall here several elementary properties of $T_{1}$-spaces which are not necessarily Hausdorff. They are well-known and rather trivial for $T_{2^{-}}$ spaces, while for $T_{1}$-ones they should also be known for specialists, thought we did not find their explicit exposition in the literature. Therefore to make the paper self-contained and for future references we will collect them together and present their short proofs. These resutls will be applied to the spaces of leaves of foliations.

Recall that a topological space $Y$ is compact if every open cover of $Y$ contains a finite subcover. In this paper a (not necessarily Hausdorff) topological space $Y$ will be called locally compact ${ }^{2}$ whenever for each $y \in Y$ and an open neighborhood $U$ of $y$ there exists a compact subset $B \subset Y$ such that $y \in \operatorname{Int}(B) \subset \bar{B} \subset U$.

2.1. Exponential law. For two sets $A$ and $B$ denote by $\operatorname{Map}(A, B)$ the set of all maps $f: A \rightarrow B$. Then for any other set $C$ we have a natural bijection

$$
E=E_{A, B, C}: \operatorname{Map}(A \times B, C) \rightarrow \operatorname{Map}(A, \operatorname{Map}(B, C))
$$

called an exponential map and defined as follows: if $F: A \times B \rightarrow C$ is a map, then $E(F): A \rightarrow \operatorname{Map}(B, C)$ is given by $E(F)(a)(b)=F(a, b)$ for all $(a, b) \in A \times B$.

If $A, B$ are topological spaces, it is natural to consider the space $\mathcal{C}^{0}(A, B)$ of continuous maps $A \rightarrow B$ instead of $\operatorname{Map}(A, B)$. We will always endow $\mathcal{C}^{0}(A, B)$ with the compact open topology. Then the variant of exponential law for topological spaces holds under additional assumptions of $A$ and $B$ :

Theorem 2.1.1. Let $A, B, C$ be any topological spaces. Then $E$ induces a continuous injective map

$$
E=E_{A, B, C}: \mathcal{C}^{0}(A \times B, C) \rightarrow \mathcal{C}^{0}\left(A, \mathcal{C}^{0}(B, C)\right)
$$

If $B$ is locally compact (not necessarily Hausdorff), then (2.1) is a continuous bijection. Moreover, if, in addition, $A$ is a $T_{3}$-space, then (2.1) is a homeomorphism.

This theorem is well known and usually formulated for Hausdorff spaces [2]. For non-Hausdorff spaces its proof is presented in [14, Lemma 3.3].

\footnotetext{
${ }^{2}$ Notice that there are several variations of this notion for non-Hausdorff spaces which coincide for Hausdorff ones.
} 
Corollary 2.1.2. If $B$ is locally compact, and $C$ is an arbitrary topological space, then the exponential map

$$
E=E_{[0,1], B, C}: \mathcal{C}^{0}([0,1] \times B, C) \rightarrow \mathcal{C}^{0}\left([0,1], \mathcal{C}^{0}(B, C)\right)
$$

is a homeomorphism. In particular, it induces a bijection

$$
E_{0}: \pi_{0} \mathcal{C}^{0}([0,1] \times B, C) \rightarrow \pi_{0} \mathcal{C}^{0}\left([0,1], \mathcal{C}^{0}(B, C)\right) .
$$

between the corresponding sets of path components.

Moreover, for groups of homeomorphisms one can say more. Let

- $\mathcal{H}(B)$ be the group of homeomorphisms of a topological space $B$;

- $\mathcal{H}_{\text {id }}(B)$ be the path component of $\operatorname{id}_{B}$ in $\mathcal{H}(B)$, so it consists of $h \in \mathcal{H}(B)$ such that there exists a continuous path $\gamma:[0,1] \rightarrow \mathcal{H}(B)$ such that $\gamma(0)=\operatorname{id}_{B}$ and $\gamma(1)=h$;

- $\widetilde{\mathcal{H}}_{\mathrm{id}}(B)$ be the subgroup of $\mathcal{H}(B)$ consisting of homeomorphisms isotopic to $\operatorname{id}_{B}$.

Corollary 2.1.3. For any topological space $B$ we have that

(1) $\widetilde{\mathcal{H}}_{\mathrm{id}}(B) \subset \mathcal{H}_{\mathrm{id}}(B)$ and these subgroups are normal in $\mathcal{H}(B)$;

(2) if $B$ is locally compact, $\widetilde{\mathcal{H}}_{\mathrm{id}}(B)=\mathcal{H}_{\mathrm{id}}(B)$;

(3) the set $\pi_{0} \mathcal{H}(B)$ of path components of $\mathcal{H}(B)$ has a group structure under which the natural map $p: \mathcal{H}(B) \rightarrow \pi_{0} \mathcal{H}(B)$ is an epimorphism with kernel $\mathcal{H}_{\mathrm{id}}(B)$, whence $p$ induces an isomorphism of groups $\mathcal{H}(B) / \mathcal{H}_{\text {id }}(B) \cong \pi_{0} \mathcal{H}(B) ;$

Proof. (1) By Theorem 2.1.1 every isotopy $F:[0,1] \times B \rightarrow B$ induces a continuous path $E(F):[0,1] \rightarrow \mathcal{H}(B)$ between $F_{0}$ and $F_{1}$. This implies that $\widetilde{\mathcal{H}}_{\text {id }}(B) \subset \mathcal{H}_{\text {id }}(B)$. Normality of these groups is easy.

(2) If $B$ is locally compact, then by Corollary 2.1.2 the exponential map $E_{[0,1], B, B}$ is a homeomorphism, so every continuous path $\gamma:[0,1] \rightarrow \mathcal{H}(B)$ is induced by some isotopy $E^{-1}(\gamma):[0,1] \times B \rightarrow B$. This gives the inverse inclusion $\widetilde{\mathcal{H}}_{\text {id }}(B) \supset \mathcal{H}_{\text {id }}(B)$.

Statement (3) is straightforward.

Thus for locally compact spaces isotopies can equally be thought of as paths in the group of homeomorphisms endowed with compact open topologies.

2.2. Special points of $T_{1}$ spaces. Let $Y$ be a topological space.

Definition 2.2.1. For a point $z \in Y$ define its Hausdorff closure, hcl $(z)$, to be the intersection of closures of all neighborhoods of $z$, that is

$$
\operatorname{hcl}(y)=\bigcap_{V \text { is a neighborhood of } y} \bar{V} \text {. }
$$


Evidently, $z \in \operatorname{hcl}(z)$. We say that $z$ is $\operatorname{special}^{3}$ whenever $\operatorname{hcl}(z) \backslash z \neq \varnothing$. Denote by $\mathcal{V}$ the set of all special points of $Y$.

One easily check that $Y$ is Hausdorff iff $\operatorname{hcl}(z)=\{z\}$ for all $z \in Y$, that is $\mathcal{V}=\varnothing$.

Further, let $L \subset Y$ be a subset, $y \in L$, and $\operatorname{hcl}_{L}(y)$ be Hausdorff closure of $z$ in $L$ with respect to the induced topology from $Y$. It is straightforward that

$$
\operatorname{hcl}_{L}(y) \subset \operatorname{hcl}(y) \cap L,
$$

however the opposite inclusion can fail.

Lemma 2.2.2. For a subset $A \subset Y$ the following statements hold true.

(1) If $A \cap \operatorname{hcl}(y)=\{y\}$ for all $y \in A$, then $A$ is Hausdorff.

(2) In particular, for every $z \in \mathcal{V}$ the following subspace

$$
A_{z}:=(A \backslash \mathcal{V}) \cup\{z\}=A \backslash(\mathcal{V} \backslash\{z\})
$$

of $Y$ is Hausdorff.

(3) If $A$ is compact, then $\bar{A} \subset A \cup \mathcal{V}$.

Proof. Statement (1) is an immediate consequence of (2.4).

(2) To prove that $B:=(L \backslash \mathcal{V}) \cup\{z\}$ is Hausdorff it suffices to verify that $\operatorname{hcl}_{B}(y)=\{y\}$ for all $y \in B$.

If $y \in L \backslash \mathcal{V}$, then $\operatorname{hcl}(y)=\{y\}$, whence $B \cap \operatorname{hcl}(y)=\{y\}$. Since $z \in \operatorname{hcl}(w)$ if and only if $w \in \operatorname{hcl}(z)$, it follows that $\operatorname{hcl}(z) \subset \mathcal{V}$. Therefore

$$
\begin{aligned}
B \cap \operatorname{hcl}(z) & =((L \backslash \mathcal{V}) \cup\{z\}) \cap \operatorname{hcl}(z)= \\
& =((L \backslash \mathcal{V}) \cap \operatorname{hcl}(z)) \cup(\{z\} \cap \operatorname{hcl}(z))=\varnothing \cup\{z\}=\{z\} .
\end{aligned}
$$

(3) Let $y \notin L \cup \mathcal{V}$. We will show that then there exists an open neighborhood $W$ of $y$ such that $L \cap W=\varnothing$. This will imply that $y \notin \mathrm{Cl}(L)$, whence $\mathrm{Cl}(L) \subset L \cup \mathcal{V}$.

Since $y$ is not a special point, i.e. $\operatorname{hcl}(y)=\{y\}$, we get from (2.3) that

$$
L \subset L \cup \mathcal{V} \subset Y \backslash\{y\}=\bigcup_{V \ni y}(Y \backslash \mathrm{Cl}(V)),
$$

where $V$ runs over all open neighborhoods of $y$. Thus

$$
\{Y \backslash \bar{V} \mid V \text { is a neighborhood of } y\}
$$

is an open cover of a compact set $L$, and so it contains a finite subcover, i.e. one can find open neighborhoods $V_{1}, \ldots, V_{m}$ of $y$ such that $L \subset \bigcup_{i=1}^{m}\left(Y \backslash \mathrm{Cl}\left(V_{i}\right)\right)$. Hence $W=V_{1} \cap \ldots \cap V_{m}$ is an open neighborhood of $y$ with $L \cap W=\varnothing$.

\footnotetext{
${ }^{3}$ In $[6,8]$ such points were called branch.
} 
Evidently, statement (3) of Lemma 2.2.2 extends the well known fact that a compact subset $A$ of a Hausdorff space $Y$ is closed.

Corollary 2.2.3. Let $A$ be a compact subset of the subspace $Y \backslash \mathcal{V}$. Then it is closed in $Y$.

Proof. Let $x \in \bar{A} \backslash A \subset \mathcal{V}$. Consider the Hausdorff subspace $A_{x}$ of $Y$. Then $A$ is closed in $A_{x}$ as a compact subset of the Hausdorff space $A_{x}$, whence $A=\bar{A} \cap A_{x}$. On the other hand $x \in \bar{A} \cap A_{x}$ by definition which is impossible.

2.3. Locally finite subsets. Say that a subset $A \subset Y$ is locally finite if for every point $z \in Y$ there exists an open neighborhood $U$ such that the intersection $U \cap A$ is finite. In other words, the family $\{\{a\} \mid a \in A\}$ of one-point subsets of $A$ is locally finite in $Y$.

Lemma 2.3.1. Consider the following conditions on a subset $A$ of a topological space $Y$ :

(1) A is closed and discrete;

(2) for each $y \in Y$ there exists a neighborhood $V$ intersecting $A$ in at most one point;

(3) A is locally finite;

Then we have the following implications: $(1) \Rightarrow(2) \Rightarrow(3)$.

If $Y$ is $T_{1}$ then we also have that $(3) \Rightarrow(1)$, i.e. all the above conditions are equivalent.

Proof. $(1) \Rightarrow(2)$. Suppose $A$ is closed and discrete and let $y \in Y$. If $y \notin A$, then $V=Y \backslash A$ is an open neighborhood of $y$ that does not intersect $A$. If $y \in A$, then discreteness of $A$ implies that there exists an open neighborhood $V$ of $y$ such that $V \cap A=\{y\}$.

The implication $(2) \Rightarrow(3)$ is evident.

It remains to prove the implication $(3) \Rightarrow(1)$ under the assumption that $Y$ is $T_{1}$. Suppose $A$ is locally finite. Then each subset $B \subset A$ is locally finite as well. Moreover, as every point $y \in Y$ is a closed subset, it follows that $B$ is closed as a union of a locally finite family of its closed one-point subsets. In other words every subset of $A$ is closed in $Y$. Hence $A$ is closed and discrete.

2.4. One-dimensional $T_{1}$ manifolds. Let $Y$ be a $T_{1}$ topological space locally homeomorphic with $[0,1)$. In other words, $Y$ is a one-dimensional manifold which is $T_{1}$ but not necessarily Hausdorff. Then $Y$ is also locally compact, and by Corollary 2.1 .3 one can equally regard $\pi_{0} \mathcal{H}(Y)$ as the 
group of path components of $\mathcal{H}(Y)$ and also as the group of isotopy classes of homeomorphisms of $Y$.

As usual, a point $y \in Y$ is called internal, if it has an open neighborhood homeomorphic with $(0,1)$. Otherwise, $y$ has an open neighborhood homeomorphic with $[0,1)$ and is called a boundary point. As usual, the sets of all internal and boundary points will be denoted by $\operatorname{Int}(Y)$ and $\partial Y$ respectively.

The following Lemma 2.4.1 characterizes the identity path component of $Y$ under assumption that the set of its special points is locally finite.

Lemma 2.4.1. Let $Y$ be a $T_{1}$ space locally homeomorphic with $[0,1)$ such that the set $\mathcal{V}$ of its special points is locally finite and every connected component of the complement $A:=Y \backslash \mathcal{V}$ is second-countable. Let also $z \in \mathcal{V}$ be any special point and $A_{z}:=A \cup\{z\}$. Then the following statements hold.

(1) $A$ and $A_{z}$ are open and Hausdorff.

(2) Every connected component $C$ of $A$ or of $A_{z}$ is open in $Y$ and is homeomorphic to one of the following spaces: the circle $S^{1},[0,1],[0,1)$, $(0,1)$. In particular, $C$ is orientable.

(3) If a connected component $C$ of $A$ is compact then it is a connected component of $Y$.

(4) Let $k \in \mathcal{H}(Y)$. Then $k \in \mathcal{H}_{\mathrm{id}}(Y)$ if and only if

(YA) $k$ is fixed on $T:=\partial Y \cup \mathcal{V}$;

(YB) $k(C)=C$ for every connected component $C$ of $Y \backslash T$, and the restriction map $\left.k\right|_{C}: C \rightarrow C$ is isotopic to $\mathrm{id}_{C}$ (which in the case of 1-dimensional manifolds is equivalent to the assumption that $\left.k\right|_{C}$ preserves orientation of $C$ ).

If these conditions hold, then $k$ is also fixed on $\partial Y$.

Proof. (1) By Lemma 2.2.2, $A$ and $A_{z}$ are Hausdorff. Moreover, as $Y$ is $T_{1}$ and $\mathcal{V}$ is locally finite, it follows that $\mathcal{V}$ and $\mathcal{V} \backslash\{z\}$ are closed in $Y$, whence their complements $A$ and $A_{z}$ are open.

(2) This statement is proved in [17, Lemma 2.3]. Let us recall the arguments. Since $A_{z}$ is open in $Y$ and $Y$ is locally path connected, it follows that every connected component $C$ of $A$ and $A_{z}$ is open in $Y$ as well. Moreover, by assumption every connected component of $A$ is second countable. As $z$ has a countable base of open neighborhoods each being a union of $z$ and at most two open subsets of $A$, it follows that the connected components of $A_{z}$ are also second countable. Hence $C$ is a connected Hausdorff 1-manifold, and the results follows from the classification of such manifolds, e.g. [10, Exercises 1.2.6 and 1.4.9]. 
(3) The connected set $C$ is both open, see (1) and closed, see Corollary 2.2.3.

(4) Necessity. Let $k \in \mathcal{H}_{\text {id }}(Y)$, so there is an isotopy $F:[0,1] \times Y \rightarrow Y$ such that $F_{0}=\operatorname{id}_{Y}$ and $F_{1}=k$. We need to check conditions (YA) and (YB) for $k$.

First notice that $\mathcal{V}$ and $\partial Y$ are defined in topological terms, i.e. they are invariant under self-homeomorphisms of $Y$. Moreover, each $z \in \partial Y \backslash \mathcal{V}$ has a neighborhood $U_{z}$ such that $U_{z} \cap T=\{z\}$. Since $\mathcal{V}$ is locally finite, and therefore discrete due to Lemma 2.3.1, we obtain that $T$ is discrete as well. This implies that $F([0,1] \times z) \subset T$ for any $z \in T$, and therefore $F([0,1] \times z)$ is contained in some connected component of $T$, being a one-point set since $T$ is discrete. Hence $F_{t}(z)=F_{0}(z)=z$ for all $t \in[0,1]$. In particular, $k$ is fixed on $T$, which proves (YA).

As $Y \backslash T$ is also invariant under self-homeomorphisms of $Y$, we obtain by similar arguments that $F([0,1] \times C) \subset C$ for every connected component $C$ of $Y \backslash T$. Thus the restriction

$$
\left.F\right|_{[0,1] \times C}:[0,1] \times C \rightarrow C
$$

is an isotopy between $\operatorname{id}_{C}$ and $\left.k\right|_{C}$. Therefore, $\left.k\right|_{C}: C \rightarrow C$ preserves orientation of $C$ which proves (YB).

Sufficiency. Let $k \in \mathcal{H}(Y)$ be a homeomorphism satisfying (YA) and (YB). For every connected component $C$ of $A=Y \backslash T$ fix an arbitrary isotopy $F^{C}:[0,1] \times C \rightarrow C$ such that $F_{0}^{C}=\operatorname{id}_{C}$ and $F_{1}^{C}=\left.k\right|_{C}$ and define the map $G:[0,1] \times Y \rightarrow Y$ by

$$
G(t, x)= \begin{cases}x, & x \in T, \\ F^{C}(t, x), & x \in C, \text { where } C \text { is a connected component of } Y \backslash T .\end{cases}
$$

Then $G_{0}=\operatorname{id}_{Y}, G_{1}=k$, and each $G_{t}$ is a bijection of $Y$. Notice that $\left.G\right|_{A \times[0,1]}$ is an isotopy of $A$, and $G$ is its extension to $Y \times[0,1]$ fixed at special points. We need to show that $G$ is an isotopy of $Y$.

For each $z \in T$ let $C_{z}$ be a connected component of $A_{z}:=A \cup\{z\}$ containing $z$. Then $A \cup\left\{C_{z}\right\}_{z \in T}$ is an open cover of $Y$. Since the restriction $\left.G\right|_{A \times[0,1]}: A \times[0,1] \rightarrow A$ is an isotopy, it suffices to show that for each $z \in T$, the restriction $G: C_{z} \times[0,1] \rightarrow C_{z}$ is an isotopy as well.

If $C_{z}$ is compact, i.e. it is homeomorphic either to $S^{1}$ or to $[0,1]$, then $C_{z}$ is a one-point compactification of $C$, and continuity of $\left.G\right|_{C_{z} \times[0,1]}$ follows from [1, Theorem 2.1].

Otherwise, $\left(C_{z}, z\right)$ is homeomorphic to one of the following pointed spaces:

$$
((-1,1), 0), \quad([-1,1), 0), \quad([0,1), 0) .
$$

In this case the proof can proceed similarly to the arguments of [1, Theorem 3.2] extending the previous case one-point compactification of $C$ to the 
case of the so-called ideal Freudental compactification. In fact, the proof of [1, Theorem 3.2] consists of proving continuity of $G$ for each added point, which in our situation is $z$ only.

Remark 2.4.2. The problem of one-point extensions of isotopies (as in the proof of continuity of $G$ ) were studied by R. H. Crowell [1, Theorem 2.1], H. Gluck [5] and many others. Also, certain variants of such results for concordancies of homeomorphisms considered in L. S. Husch and T. B. Rushing [11], applications to ambient isotopies of arcs in manifolds [4,22], and applications to Nielsen numbers are given in P. Heath and X. Zhao $[9, \S 4]$.

\section{STRIPED SURFACES}

In this section we will briefly recall the results by W. Kaplan, S. Maksymenko, E. Polulyakh, and Yu. Soroka.

3.1. Quasi-striped surfaces. The following notion of a quasi-strip is a "basic block" generating foliations on the plane.

Definition 3.1.1. A foliated surface $\left(S, \Delta_{S}\right)$ will be called a quasi-strip whenever

a) every boundary component of $S$ is an open arc being also a leaf of $\Delta_{S}$;

b) the restriction of $\Delta_{S}$ to the interior $\operatorname{Int}(S)$ is foliated homeomorphic with $\mathbb{R}^{2}$ endowed by trivial foliation into horizontal lines $\{\mathbb{R} \times y\}_{y \in \mathbb{R}}$.

Definition 3.1.2. Let $\Delta=\{\mathbb{R} \times y\}_{y \in[0,1]}$ be the trivial foliation on $\mathbb{R} \times[0,1]$ into horizontal lines. Then for every open (in the induced topology) subset $S \subset \mathbb{R} \times[0,1]$ such that $\mathbb{R} \times(0,1) \subset S$ the foliated surface $\left(S,\left.\Delta\right|_{S}\right)$ will be called a model strip. In this case we will use the following notation:

$$
\begin{aligned}
\partial_{0} S & :=S \cap(\mathbb{R} \times\{0\}), & \partial_{1} S & :=S \cap(\mathbb{R} \times\{1\}), \\
\partial S & :=\partial_{0} S \cup \partial_{1} S, & \operatorname{Int}(S) & :=\mathbb{R} \times(0,1),
\end{aligned}
$$

and call $\partial S$ the boundary of $S$, and $\partial_{0} S$ and $\partial_{1} S$ the boundary sides of $S$.

Thus a model strip is a union of $\mathbb{R} \times(0,1)$ with at most countably many (possibly zero) open mutually disjoint intervals contained in $\mathbb{R} \times\{0,1\}$. Evidently, each model strip is a quasi-strip as well. The difference between these two notions is that in the model strip all boundary components are distributed between two boundary sides only, while quasi-strips may have boundary components between internal leaves.

Definition 3.1.3. Let $Z$ be a two-dimensional topological manifold (a surface) and $Z_{0}=\bigsqcup_{\lambda \in \Lambda} S_{\lambda}$ be a family of mutually disjoint quasi-strips $\left\{S_{\lambda}\right\}_{\lambda \in \Lambda}$. A quasi-striped atlas on $Z$ is a map $q: Z_{0} \rightarrow Z$ such that 
1) $q$ is a quotient map, i.e. it is continuous, surjective, and a subset $U \subset Z$ is open if and only if $q^{-1}(U) \cap S_{\lambda}$ is open in $S_{\lambda}$ for each $\lambda \in \Lambda$;

2) there exist two disjoint families $\mathcal{X}=\left\{X_{j}\right\}_{j \in \Gamma}$ and $\mathcal{Y}=\left\{Y_{j}\right\}_{j \in \Gamma}$ of mutually distinct boundary intervals of $Z_{0}$ enumerated by the same set of indexes $\Gamma$ such that

(a) $q$ is injective on $Z_{0} \backslash(\mathcal{X} \cup \mathcal{Y})$;

(b) $q\left(X_{j}\right)=q\left(Y_{j}\right)$ for each $j \in \Gamma$, and the restrictions

$$
X_{j} \stackrel{\left.q\right|_{X_{j}}}{\longrightarrow} q\left(X_{j}\right)=q\left(Y_{j}\right) \stackrel{\left.q\right|_{Y_{j}}}{\longleftarrow} Y_{j}
$$

are bijections.

The images $q\left(X_{j}\right)=q\left(Y_{j}\right), j \in \Gamma$, will be called seams of $Z$ (as well as of the striped atlas $q)$.

If all strips $\left\{S_{\lambda}\right\}_{\lambda \in \Lambda}$ are model, then the quasi-striped atlas $q$ will be called striped.

Condition (b) implies that for each $j \in \Gamma$ we have a well-defined "gluing" homeomorphism $\phi_{j}=\left.\left(\left.q\right|_{X_{j}}\right)^{-1} \circ q\right|_{Y_{j}}: Y_{j} \rightarrow X_{j}$. Thus a quasi-striped surface is obtained from a family of quasi-strips by gluing them along certain pairs of boundary intervals by homeomorphisms $\phi_{j}$. It is allowed to glue two quasi-strips along more than one pair of boundary components, and one may also glue boundary components belonging to the same quasi-strip.

Notice that the foliations $\Delta_{S_{\lambda}}$ on strips $S_{\lambda}$ give a unique foliation $\Delta_{0}$ on $Z_{0}$. In this case if $\gamma$ is a leaf of $\Delta_{0}$ contained in a strip $S_{\lambda}$, then we will say that $S_{\lambda}$ is a strip of $\gamma$.

Also, the images of leaves of $\Delta_{0}$ under $q$ constitute a one-dimensional foliation $\Delta$ on $Z$ which we will call the canonical foliation associated with the quasi-striped atlas $q$.

Definition 3.1.4. A foliated surface $(Z, \Delta)$ will be called a (quasi-)striped surface whenever it admits a (quasi-)striped atlas whose canonical foliation is $\Delta$.

Now the results by W. Kaplan can be formulated as follows.

Theorem 3.1.5 (W. Kaplan $[12,13])$. Every one-dimensional foliation $\Delta$ on the plane $\mathbb{R}^{2}$ belongs to the class $\mathcal{F}$ and admits a quasi-striped atlas, i.e. $\Delta$ is a canonical foliation associated with some quasi-striped atlas $q$ on $\mathbb{R}^{2}$.

Remark 3.1.6. Let $(Z, \Delta)$ be any (oriented or non-oriented, compact or non-compact) connected surface distinct from $S^{2}$ and $\mathbb{R} P^{2}$. Then the universal covering of its interior $\operatorname{Int}(Z)$ is homeomorphic with $\mathbb{R}^{2}$, e.g. $[3$, Corollary 1.8]. Moreover, if $p: \mathbb{R}^{2} \rightarrow \operatorname{Int}(Z)$ is the corresponding universal 
covering map, then one has a foliation $F$ on $\mathbb{R}^{2}$ induced from $\Delta$ : the leaves of $F$ are connected components of the inverse images $p^{-1}(\omega), \omega \in \Delta$. There is also a natural free action of the fundamental group $\pi_{1} Z$ on $\mathbb{R}^{2}$ by foliated homeomorphisms so that $\left(\operatorname{Int}(Z),\left.\Delta\right|_{\operatorname{Int}(Z)}\right)$ is the quotient $\left(\mathbb{R}^{2}, F\right) / \pi_{1} Z$.

This observation shows that foliations on $\mathbb{R}^{2}$ determine all foliations on all connected boundaryless surfaces distinct from $S^{2}$ and $\mathbb{R} P^{2}$. However, this does not help so much, since usually it is hard to explicitly describe the action of $\pi_{1} Z$ of $\mathbb{R}^{2}$ by covering transformations.

Also, due to Theorem 3.1.5 the foliated surface $\left(\mathbb{R}^{2}, F\right)$ admits a quasistriped atlas. However, again such a splitting into quasi-strips in general is not related with the action of $\pi_{1} Z$. Therefore it is still better to work with the surface $Z$ instead of $\mathbb{R}^{2}$.

In fact, the splitting of a quasi-striped surface into quasi-strips proposed by Kaplan in Theorem 3.1.5 is not unique. This led the present authors to consider quasi-striped surfaces glued from model strips only, and it turned out that splitting into such strips (if it exists) is unique, see Theorem 3.1.10.

Remark 3.1.7. Let us discuss distinct types of leaves of $\Delta$.

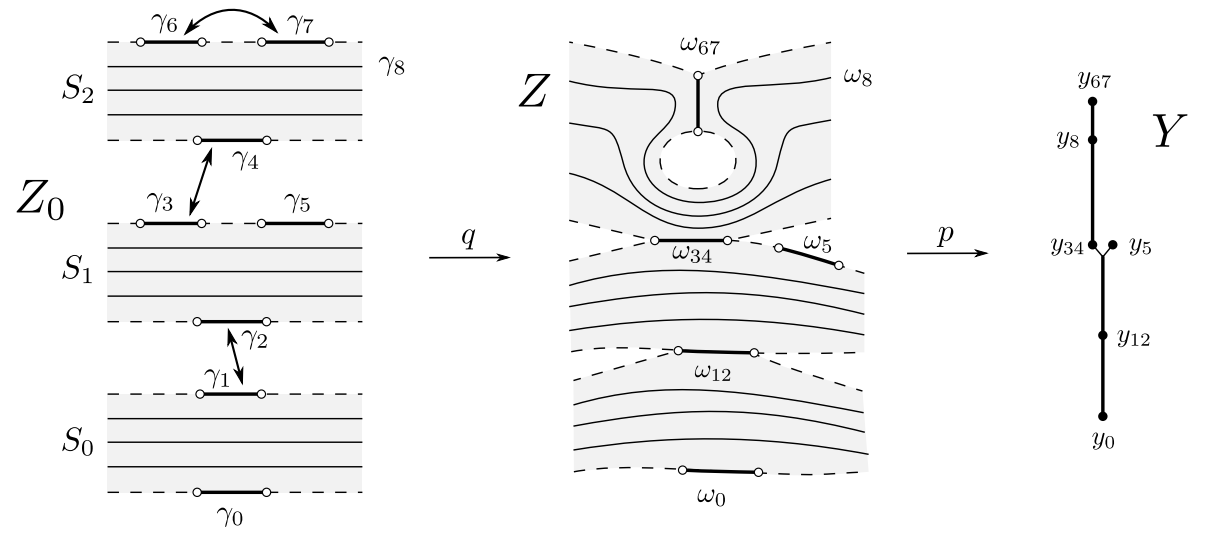

FIGURE 3.1.

1) For each leaf $\gamma=\mathbb{R} \times\{t\} \subset \operatorname{Int}\left(S_{\lambda}\right), t \in(0,1)$,

$$
\omega:=q(\gamma) \in \Delta_{r e g}, \quad y:=p(\omega) \in \operatorname{Int}(Y) \backslash \mathcal{V},
$$

see the leaf $\omega_{8}$ in Figure 3.1.

2) Moreover, suppose $\omega \in \Delta$ is a seam, so $q^{-1}(\omega)=X_{j} \cup Y_{j}$, where $X_{j} \subset \partial_{\varepsilon} S_{\lambda}$, and $Y_{j} \subset \partial_{\varepsilon^{\prime}} S_{\lambda^{\prime}}$ for some $\varepsilon, \varepsilon^{\prime} \in\{0,1\}$ and $\lambda, \lambda^{\prime} \in \Lambda$. Then the following characterizations hold.

a) $\omega \in \Delta_{r e g}$ if and only if $X_{j}=\partial_{\varepsilon} S_{\lambda}$ and $Y_{j}=\partial_{\varepsilon^{\prime}} S_{\lambda^{\prime}}$, see leaf $\omega_{12}$ in Figure 3.1. 
b) $\omega \in \Delta_{\text {sing }} \backslash \Delta_{\text {spec }}$ if and only if $\lambda=\lambda^{\prime}, \varepsilon=\varepsilon^{\prime}$, and $\partial_{\varepsilon} S_{\lambda}=X_{j} \cup Y_{j}$. In other words, $\partial_{\varepsilon} S_{\lambda}$ consists of two boundary intervals, and $\omega$ is obtained by gluing those inervals. In this case $y:=p(\omega) \in \partial Y \backslash \mathcal{V}$, so $Y$ is Hausdorff at $y$, see the leaf $\omega_{67}$ in Figure 3.1.

c) In all other cases, $\omega \in \Delta_{\text {spec }}$, and hcl $(\omega)$ contains all leaves from

$$
q\left(\partial_{\varepsilon} S_{\lambda} \cup \partial_{\varepsilon^{\prime}} S_{\lambda^{\prime}}\right)
$$

see leaves $\omega_{34}$ and $\omega_{5}$ in Figure 3.1.

Notice that in the case a) one can replace $S_{\lambda} \cup S_{\lambda^{\prime}}$ with a single strip, and get another striped atlas for $(Z, \Delta)$ having one less strip. This leads to the following notion.

A striped atlas will be called reduced whenever

$$
q\left(\partial Z_{0}\right)=\partial Z \cup \Delta_{\text {sing }} .
$$

The following statement is easy and we leave it for the reader.

Lemma 3.1.8 (cf. [17, Lemma 2.1]). Let $q: Z_{0} \rightarrow Z$ be a striped atlas, with $\Delta$ the corresponding canonical foliation on $Z, p: Z \rightarrow Y$ the projection to the space of leaves, and $\mathcal{V}$ the set of special points of $Y$. Then

$$
p(\operatorname{hcl}(\omega))=\operatorname{hcl}(p(\omega))
$$

for every leaf $\omega \in \Delta$. Moreover,

$$
p\left(\Delta_{\text {spec }}\right)=\mathcal{V}, \quad p\left(\Delta_{\text {sing }} \backslash \Delta_{\text {spec }}\right) \subset \partial Y \backslash \mathcal{V}, \quad p\left(\partial Z \cup \Delta_{\text {sing }}\right)=\partial Y \cup \mathcal{V} .
$$

If $q$ is reduced, then for every connected component $C$ of $Y \backslash(\partial Y \cup \mathcal{V})$, $q^{-1}\left(p^{-1}(C)\right)$ is an interior of some model strip $S_{\lambda} \subset Z_{0}$. Define the following path $\gamma_{C}:(0,1) \rightarrow \operatorname{Int}\left(S_{\lambda}\right)=\mathbb{R} \times(0,1)$ by $\gamma_{C}(t)=(0, t), t \in(0,1)$. Then the composition $p \circ q \circ \gamma_{C}:(0,1) \rightarrow C$ is a homeomorphism.

Theorem 3.1.9 ([20, Theorem 4.4]). Let $(Z, \Delta)$ be a foliated surface from class $\mathcal{F}$. Then the following conditions are equivalent:

(A4) the family $\Delta_{\text {sing }}$ of all singular leaves of $\Delta$ is locally finite;

(A5) $(Z, \Delta)$ is a striped surface.

Theorem 3.1.10 ([16, Theorem 3.7]). Every connected striped surface $Z$ with countable base is foliated homeomorphic either to

(1) an open cylinder $C$ or an open Möbius band $M$ foliated by fibers of one-dimensional vector bundles $C \rightarrow S^{1}$ and $M \rightarrow S^{1}$ over the circle, or to

(2) a striped surface with reduced atlas. 
Notice that in the case (1) all leaves of $\Delta$ are regular. Also in the case (2) the seams are precisely the singular leaves contained $\operatorname{in} \operatorname{Int}(Z)$ and thus we have a canonical decomposition of $Z$ into strips.

\subsection{Characterization of the homeotopy group $\mathcal{H}_{\mathrm{id}}(Z, \Delta)$ for striped} surfaces. Let $q: Z_{0} \rightarrow Z$ be a striped atlas, and $h \in \mathcal{H}(Z, \Delta)$ a homeomorphism such that $h\left(q\left(S_{\lambda}\right)\right)=q\left(S_{\lambda}\right)$ for some $\lambda \in \Lambda$. Since $q$ is injective on the interior of $S_{\lambda}$, as well as on each boundary component of $S_{\lambda}$, it follows that there exists a unique foliated homeomorphism $h_{0}: S_{\lambda} \rightarrow S_{\lambda}$ making commutative the following diagram:

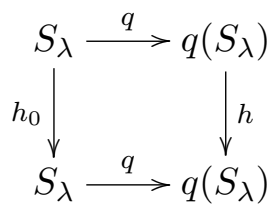

Moreover, see [16, Lemma 4.1], $h_{0}(x, y)=(\alpha(x, y), \beta(y))$, where

- $\beta:[0,1] \rightarrow[0,1]$ is a homeomorphism;

- for each $y \in(0,1)$ the map $\alpha_{y}: \mathbb{R} \rightarrow \mathbb{R}, \alpha_{y}(x)=\alpha(x, y), x \in \mathbb{R}$, is a homeomorphism;

- for each $y \in\{0,1\}$ the map $\alpha_{y}: \partial_{y} S_{\lambda} \rightarrow \partial_{\beta(y)} S_{\lambda}, \alpha_{y}(x)=\alpha(x, y)$, is a monotone homeomorphism between linearly ordered subsets (being unions of mutually disjoint open intervals) $\partial_{y} S_{\lambda}$ and $\partial_{\beta(y)} S_{\lambda}$.

Theorem 3.2.1 ([16, Theorem 4.4]). Let $q: Z_{0} \rightarrow Z$ be a reduced striped atlas such that the family $\Delta_{\text {spec }}$ of all special leaves of the canonical foliation $\Delta$ is locally finite. Let also $h \in \mathcal{H}(Z, \Delta)$. Then $h \in \mathcal{H}_{\mathrm{id}}(Z, \Delta)$ iff the following conditions hold:

(ZA) $h$ preserves each leaf $\omega \in \partial Z \cup \Delta_{\text {sing }}$ with its orientation;

(ZB) $h\left(q\left(S_{\lambda}\right)\right)=q\left(S_{\lambda}\right)$ for all $\lambda \in \Lambda$, and if $h_{0}=(\alpha, \beta): S_{\lambda} \rightarrow S_{\lambda}$ is a unique lifting of $\left.h\right|_{S_{\lambda}}$, see $(3.2)$, then $\beta$ and each $\alpha_{y}, y \in[0,1]$, is strictly increasing.

\section{Proof of Theorem 1.4}

Let $(Z, \Delta)$ be a connected foliated surface from class $\mathcal{F}$ satisfying condition (A4). By Theorem 3.1.9, the latter condition is equivalent to the assumption (A5) that $(Z, \Delta)$ is a striped surface, i.e. there exists a striped atlas $q: Z_{0} \rightarrow Z$ such that $\Delta$ is its canonical foliation. We need to prove that $\psi\left(\mathcal{H}_{\text {id }}(Z, \Delta)\right)=\mathcal{H}_{\text {id }}(Y)$ and that the kernel of the induced homomorphism $\psi_{0}: \pi_{0} \mathcal{H}_{\mathrm{id}}(Z, \Delta) \rightarrow \mathcal{H}_{\mathrm{id}}(Y)$ is either trivial or isomorphic to $\mathbb{Z}_{2}$.

First suppose that $Z$ is connected. Then due to Theorem 3.1 .10 we have the following three cases. 
1) $Z=\mathbb{R} \times S^{1}$ is an open cylinder, $Y=S^{1}$ is the circle, $p: \mathbb{R} \times S^{1} \rightarrow S^{1}$ is given by $p(x, y)=y$, and $\Delta$ consists of lines $\mathbb{R} \times\{y\}, y \in S^{1}$. Then every $h \in \mathcal{H}(Z, \Delta)$ is given by $h(x, y)=\left(\alpha_{h}(x, y), \beta_{h}(y)\right)$, where $\beta_{h}: S^{1} \rightarrow S^{1}$ is some homeomorphism, and $\alpha_{h}: \mathbb{R} \times S^{1} \rightarrow \mathbb{R}$ is a continuous map such that the correspondence $x \mapsto \alpha_{h}(x, y)$ is a homeomorphism $\mathbb{R} \rightarrow \mathbb{R}$ which preserves or reverses orientation mutually for all $y \in S^{1}$. In particular, we have a homomorphism or : $\mathcal{H}(Z, \Delta) \rightarrow \mathbb{Z}_{2} \oplus \mathbb{Z}_{2}$, or $(h)=\left(\operatorname{or}\left(\alpha_{h}\right)\right.$, or $\left.\left(\beta_{h}\right)\right)$, where $\operatorname{or}\left(\beta_{h}\right)=0$ if $\beta_{h}$ preserves orientation, or $\left(\beta_{h}\right)=1$ otherwise, and similarly for $\alpha_{h}$.

Evidently $\psi(h)=\beta_{h}$. This also implies that if $\beta \in \mathcal{H}(Y)$ and $h \in \mathcal{H}(Z, \Delta)$ is given by $h(x, y)=(x, \beta(y))$, then $\psi(h)=\beta$. Hence $\psi$ is surjective.

It is well-known that $\mathcal{H}_{\text {id }}(Y)$ consists of homeomorphisms preserving orientation of $Y$. Also, see e.g. [21, Examples 6.5], one easily checks that $h \in \mathcal{H}_{\text {id }}(Z, \Delta)$ iff $\beta_{h}$ and the correspondences $x \mapsto \alpha_{h}(x, y)$ preserve orientations. In particular, we get the following commutative diagram:

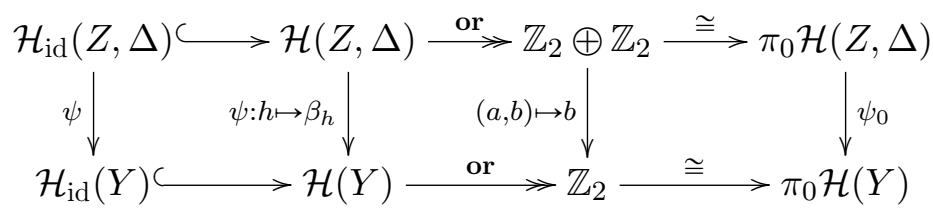

This implies Theorem 1.4 for this case.

2) The proof of Theorem 1.4 for the case when $Z$ is an open Möbius band and $p: Z \rightarrow S^{1}$ is a locally trivial fibration with fiber $\mathbb{R}$ is similar, and we leave if to the reader.

3) In all other cases due to Theorem 3.1 .10 one can assume that $q$ is a reduced striped atlas.

a) First we prove that $\psi\left(\mathcal{H}_{\text {id }}(Z, \Delta)\right) \subset \mathcal{H}_{\text {id }}(Y)$ which will imply that $\psi$ induces a homomorphism $\psi_{0}: \pi_{0} \mathcal{H}_{\mathrm{id}}(Z, \Delta) \rightarrow \pi_{0} \mathcal{H}_{\mathrm{id}}(Y)$.

Let $h \in \mathcal{H}_{\mathrm{id}}(Z, \Delta)$ and $k=\psi(k)$. Thus $h$ satisfies conditions (ZA) and (ZB) of Theorem 3.2.1, and we should check that $k \in \mathcal{H}_{\mathrm{id}}(Y)$, i.e. it satisfies conditions (YA) and (YB) of Lemma 2.4.1.

Due to (ZA), $h$ preserves each leaf $\omega \subset \partial Z \cup \Delta_{\text {sing }}$, whence $k$ fixes each point $y \in \partial Y \cup \mathcal{V}=p\left(\partial Z \cup \Delta_{\text {sing }}\right)$, see Lemma 3.1.8, i.e. $k$ satisfies condition (YA).

Furthermore, let $C$ be a connected component of $Y \backslash(\partial Y \cup \mathcal{V})$. Then by Lemma 3.1.8, $q^{-1}\left(p^{-1}(C)\right)$ is the interior of some model strip $S_{\lambda}$. Moreover, by property (ZB), $h\left(q\left(S_{\lambda}\right)\right)=q\left(S_{\lambda}\right)$, and $h$ lifts to a homeomorphism $h_{0}=(\beta, \alpha): S_{\lambda} \rightarrow S_{\lambda}$ in which $\beta$ is strictly increasing. But the latter is equivalent to the assumption that $k$ preserves orientation of $C$, i.e. satisfies condition (YB).

b) Let us show that $\psi\left(\mathcal{H}_{\text {id }}(Z, \Delta)\right)=\mathcal{H}_{\text {id }}(Y)$. 
Let $k \in \mathcal{H}_{\text {id }}(Y)$. We should find $h \in \mathcal{H}_{\text {id }}(Z, \Delta)$ such that $\psi(h)=k$. In fact we will define a homeomorphism $h_{0}: Z_{0} \rightarrow Z_{0}$ which will induce the desired $h$.

Let $C$ be a connected component of $Y \backslash T$, so $r^{-1}(C)$ is the interior of some model strip $S_{\lambda}$. Then by Lemma 3.1.8, the map $\gamma:(0,1) \rightarrow C$, $\gamma(y)=r(0, y), y \in(0,1)$, is a homeomorphism. Since by (YB), $k(C)=C$ and $k$ preserves its orientation, the map

$$
\beta=\gamma^{-1} \circ k \circ \gamma:(0,1) \rightarrow(0,1)
$$

is an orientation preserving homeomorphism of $(0,1)$. Therefore $\beta$ extends to a self-homeomorphism $\beta:[0,1] \rightarrow[0,1]$ by setting $\beta(0)=0$ and $\beta(1)=1$, and we define $h_{0}: S_{\lambda} \rightarrow S_{\lambda}$ by $h_{0}(x, y)=(x, \beta(y))$, so it is also fixed on $S_{\lambda}$.

It now follows that $h_{0}$ induces a homeomorphism $h \in \mathcal{H}_{\text {id }}(Z, \Delta)$ fixed on $\partial Z \cup \Delta_{\text {sing }}$ and such that $h_{0} \circ q=q \circ h$. Then $h$ in turn induces a homeomorphism $k^{\prime}=\psi(h): Y \rightarrow Y$ fixed on $p\left(\partial Z \cup \Delta_{\text {sing }}\right)=\partial Y \cup \mathcal{V}$ and coinciding with $k$ on the complement $Y \backslash(\partial Y \cup \mathcal{V})$. But due to (YA), $k$ is also fixed on $\partial Y \cup \mathcal{V}$, whence $k=k^{\prime}=\psi(h)$.

c) Denote $\mathcal{Q}:=\psi^{-1}\left(\mathcal{H}_{\text {id }}(Y)\right)$. Then

$$
\begin{aligned}
\operatorname{ker}\left[\psi_{0}: \pi_{0} \mathcal{H}(Z, \Delta) \rightarrow \pi_{0} \mathcal{H}(Y)\right]=\operatorname{ker}\left[\psi_{0}: \frac{\mathcal{H}(Z, \Delta)}{\mathcal{H}_{\mathrm{id}}(Z, \Delta)} \rightarrow \frac{\mathcal{H}(Y)}{\mathcal{H}_{\mathrm{id}}(Y)}\right] \cong \\
\cong \frac{\psi^{-1}\left(\mathcal{H}_{\mathrm{id}}(Y)\right)}{\mathcal{H}_{\mathrm{id}}(Z, \Delta)}=\mathcal{Q} / \mathcal{H}_{\mathrm{id}}(Z, \Delta) .
\end{aligned}
$$

Hence it remains show that $\mathcal{Q} / \mathcal{H}_{\mathrm{id}}(Z, \Delta)$ is either trivial or isomorphic with $\mathbb{Z}_{2}$.

Let $h \in \mathcal{Q}, k=\psi(h) \in \mathcal{H}_{\text {id }}(Y)$, and $h_{0}: Z_{0} \rightarrow Z_{0}$ be its unique lifting. Then $h_{0}$ preserves every boundary component of $Z_{0}$ and $h_{0}\left(S_{\lambda}\right)=S_{\lambda}$ for all $\lambda \in \Lambda$. Moreover, $h_{0}: \operatorname{Int}\left(S_{\lambda}\right)=\mathbb{R} \times(0,1) \rightarrow \mathbb{R} \times(0,1)=\operatorname{Int}\left(S_{\lambda}\right)$ is given by $h_{0}(x, y)=\left(\alpha^{\lambda}(x, y), \beta^{\lambda}(y)\right)$, where $\beta^{\lambda}$ is increasing, while $\alpha_{y}^{\lambda}$ is either increasing or decreasing mutually for all $y \in(0,1)$.

Therefore one can associate to each $\lambda$ an element or $_{h}(\lambda) \in \mathbb{Z}_{2}$ so that or $_{h}(\lambda)=0$ (resp. $\left.\mathbf{o r}_{h}(\lambda)=1\right)$ if all $\alpha_{y}^{\lambda}$ are increasing (resp. decreasing).

Note that if two strips $S_{\lambda}$ and $S_{\lambda^{\prime}}$ are glued along some of their boundary components, then $\mathbf{o r}_{h}(\lambda)=\mathbf{o r}_{h}\left(\lambda^{\prime}\right)$, whence $\mathbf{o r}_{h}$ is constant on the connected components of $Z$.

But $Z$ is connected, whence or $_{h}$ is constant. Therefore, one can associate to each $h \in \mathcal{Q}$ a well-defined element or $_{h} \in \mathbb{Z}_{2}$. It is easy to checks that the correspondence $h \mapsto$ or $_{h}$ is a homomorphism or $: \mathcal{Q} \rightarrow \mathbb{Z}_{2}$. Finally, $h \in \operatorname{ker}\left(\right.$ or) iff $h$ satisfies conditions (ZA) and (ZB), i.e. $h \in \mathcal{H}_{\text {id }}(Z, \Delta)$. 
Therefore $\operatorname{ker}\left(\psi_{0}\right)=\mathcal{Q} / \mathcal{H}_{\text {id }}(Z, \Delta)$ is either trivial or $\mathbb{Z}_{2}$. This completes Theorem 1.4.

\section{REFERENCES}

[1] R. H. Crowell. Invertible isotopies. Proc. Amer. Math. Soc., 14:658-664, 1963, doi: $10.2307 / 2034296$.

[2] R. Engelking. General topology, volume 6 of Sigma Series in Pure Mathematics. Heldermann Verlag, Berlin, second edition, 1989.

[3] D. B. A. Epstein. Curves on 2-manifolds and isotopies. Acta Math., 115:83-107, 1966, doi: $10.1007 / \mathrm{BF} 02392203$.

[4] C. D. Feustel. Homotopic arcs are isotopic. Proc. Amer. Math. Soc., 17:891-896, 1966, doi: $10.2307 / 2036278$.

[5] H. Gluck. Restriction of isotopies. Bull. Amer. Math. Soc., 69:78-82, 1963, doi: 10.1090/S0002-9904-1963-10867-8.

[6] C. Godbillon, G. Reeb. Fibrés sur le branchement simple. Enseignement Math. (2), 12:277-287, 1966 .

[7] Claude Godbillon. Fibrés en droites et feuilletages du plan. Enseign. Math. (2), 18:213224 (1973), 1972, doi: 10.5169/seals-45373.

[8] A. Haefliger, G. Reeb. Variétés (non séparées) à une dimension et structures feuilletées du plan. Enseignement Math. (2), 3:107-125, 1957.

[9] P. R. Heath, X. Zhao. Nielsen numbers for based maps, and for noncompact spaces. Topology Appl., 79(2):101-119, 1997, doi: 10.1016/S0166-8641(96)00171-X.

[10] M. W. Hirsch. Differential topology, volume 33 of Graduate Texts in Mathematics. Springer-Verlag, New York, 1994. Corrected reprint of the 1976 original.

[11] L. S. Husch, T. B. Rushing. Restrictions of isotopies and concordances. Michigan Math. J., 16:303-307, 1969, doi: 10.1307/mmj/1029000313.

[12] W. Kaplan. Regular curve-families filling the plane, I. Duke Math. J., 7:154-185, 1940, doi: 10.1215/S0012-7094-40-00710-4.

[13] W. Kaplan. Regular curve-families filling the plane, II. Duke Math J., 8:11-46, 1941, doi: 10.1215/S0012-7094-41-00802-5.

[14] O. Khokhliuk, S. Maksymenko. Smooth approximations and their applications to homotopy types. Proc. Int. Geom. Cent., 13(2):68-108, 2020, doi: 10.15673/tmgc.v13i2.1781.

[15] S. Maksymenko, O. Nikitchenko. Fundamental groupoids and homotopy types of noncompact surfaces. In Proceedings of the Winter School \&3 Workshop Wisla 20-21: Groups, invariants, integrals, and moving frames. 2022, https://arxiv.org/abs/2104. 13683. To appear.

[16] S. Maksymenko, E. Polulyakh. Foliations with non-compact leaves on surfaces. Proceedings of Geometric Center, 8(3-4):17-30, 2015.

[17] S. Maksymenko, E. Polulyakh. Foliations with all non-closed leaves on non-compact surfaces. Methods Funct. Anal. Topology, 22(3):266-282, 2016.

[18] S. Maksymenko, E. Polulyakh. One-dimensional foliations on topological manifolds. Proceedings of Geometric Center, 9(2):1-23, 2016.

[19] S. Maksymenko, E. Polulyakh. Characterization of striped surfaces. Proceedings of the International Geometry Center, 10(2):24-38, 2017.

[20] S. Maksymenko, E. Polulyakh. Characterization of striped surfaces. Proc. Int. Geom. Cent., 10(2):24-38, 2017. 
[21] S. Maksymenko, E. Polulyakh, Yu. Soroka. Homeotopy groups of one-dimensional foliations on surfaces. Proceedings of the International Geometry Center, 10(1):22-46, 2017.

[22] J. Martin, D. Rolfsen. Homotopic arcs are isotopic. Proc. Amer. Math. Soc., 19:12901292, 1968, doi: 10.2307/2036198.

[23] Yu. Soroka. Homeotopy groups of rooted tree like non-singular foliations on the plane. Methods Funct. Anal. Topology, 22(3):283-294, 2016.

[24] Yu. Soroka. Homeotopy groups of nonsingular foliations of the plane. Ukraïn. Mat. Zh., 69(7):1000-1008, 2017, doi: 10.1007/s11253-017-1423-6 (in ukrainian).

Received: September 18, 2021, accepted: November 14, 2021.

Sergiy Maksymenko

Institute of Mathematics of NAS of Ukraine, Tereshchenkivska Str. 3, Kyiv, 01024, UKRAINE

Email: maks@imath.kiev.ua

ORCID: 0000-0002-0062-5188

Eugene Polulyakh

Institute of Mathematics of NAS of Ukraine, Tereshchenkivska Str. 3, Kyiv, 01024, UKRAINE

Email: polulyah@imath.kiev.ua 